\title{
DESARROLLO DEL TALENTO HUMANO \\ PARA LÍDERES DE LA GERENCIA \\ FINANCIERA DEL SERVICIO INTEGRADO \\ DE ADMINISTRACIÓN TRIBUTARIA
}

Andrés Jerónimo Arenas Falótico(a)
Jessica Bayón Pérez(b)

DEVELOPMENT OF HUMAN TALENT IN FINANCIAL MANAGEMENT

LEADERS AT THE INTEGRATED TAX ADMINISTRATION SERVICE

DESENVOLVIMENTO DO TALENTO HUMANO PARA LÍDERES DA GERÊNCIA

FINANCEIRA DO SERVIÇO INTEGRADO DE A DMINISTRAÇÃO TRIBUTÁRIA

Fecha de recepción: 18 de mayo del 2020

Fecha de aprobación: 6 de julio del 2020

Disponible en línea: 25 de septiembre del 2020

Sugerencia de citación:

Arenas Falótico, A. J.\& Bayón Pérez, J. (2021). Desarrollo del talento humano para líderes de la gerencia financiera del servicio integrado de administración tributaria.Razón Crítica, 10, 275-293. https://doi.org/10.21789/25007807.1682

(a) Andrés Jerónimo Arenas Falótico

Doctor en Gestión, Máster en Administración de Empresas, Máster en Educación.

Profesor Universidad de Nebrija, España

https://orcid.org/0000-0002-2763-9707

aarenas@nebrija.es

(b) Jessica Bayón Pérez

Doctora en Ciencias Sociales con especialidad en Recursos Humanos. Galardonada con la Medalla de Oro al Mérito del Trabajo por la Asociación Europea de Economía y Competitividad.

Profesora de la Universidad de Nebrija, España

https://orcid.org/0000-0003-4019-7605

jbayon@nebrija.es 


\section{R E S U M E N}

El estudio tiene por propósito el desarrollo del talento humano para líderes de la gerencia financiera del servicio integrado de administración tributaria. El objetivo es el desarrollo del talento humano, por medio de los líderes de gerencia en la administración tributaria dentro del cambio organizacional. Se busca concientizar a nivel empresarial, sobre la optimización del tiempo y de los recursos, así como del empleado en función de las competencias identificadas en su puesto de trabajo. La metodología utilizada fue un diseño evaluativo, apoyado en un diseño no experimental, investigación de campo a nivel descriptivo, evaluativo ex-post y de corte cuantitativo. La técnica de recolección de datos empleada fue la encuesta, mediante un cuestionario con escala Likert que se aplicó a la población de 29 representantes de la administración tributaria en Madrid, España. La gestión por competencias pasa a transformarse en un canal comunicación entre los trabajadores y la institución; es ahora cuando en la organización comienza la formación de liderazgo en sus trabajadores, con el fin de desarrollar, optimizar y ofrecer una formación continua del personal capaz de generar conocimientos, así como estimular habilidades y destrezas en cada trabajador.

PALABRAS CLAVE: desarrollo; líderes gerenciales; talento humano. 


\section{A B S T R A C T}

This study intends to develop human talent abilities of leaders in charge of the financial management of the Integrated Tax Administration Service. The main objective is to foster the development of human talent as part of organizational change processes, increasing awareness at the business level about the optimization of time, resources and employees based on the skills identified in work positions. The methodology included an evaluative design supported by a non-experimental design, field research at a descriptive and ex-post evaluative level, and a quantitative approach. Surveys were used for data collection, applying a Likert scale-type questionnaire to a population of 29 representatives of the tax administration in Madrid, Spain. Management by competencies becomes a communication channel between workers and the institution, which makes the organization provide leadership training for its staff in order to develop, optimize and offer continuous training, thus promoting the creation of knowledge and stimulating skills and abilities in each worker.

KEYWORDS: development; managerial leaders; human talent.

\section{R E S U M O}

O objetivo deste estudo é desenvolver o talento humano para líderes da gerência financeira do Serviço Integrado de Administração Tributária (Madri, Espanha). O objetivo é o desenvolvimento do talento humano, por meio dos líderes de gerência, na administração tributária na mudança organizacional. Busca-se conscientizar, no âmbito empresarial, sobre a otimização do tempo e dos recursos, bem como do empregado em função das competências identificadas em seu lugar de trabalho. A metodologia utilizada foi um desenho avaliativo, apoiado em um desenho não experimental, pesquisa de campo no âmbito descritivo, avaliativo ex post $\mathrm{e}$ de corte quantitativo. A técnica de coleta de dados utilizada foi a pesquisa, mediante um questionário com a escala Likert que foi aplicado a uma população de 29 representantes da administração tributária em Madri. A gestão por competências passa a se transformar em um canal de comunicação entre os trabalhadores e a instituição. Nesse momento, a formação de liderança em seus trabalhadores na organização começa, a fim de desenvolver, otimizar e oferecer uma formação contínua do pessoal capaz de gerar conhecimentos e estimular habilidades e destrezas em cada trabalhador.

PALAVRAS-CHAVE: desenvolvimento; líderes gerenciais; talento humano. 


\section{N T R O D U C C I Ó N}

Con la llegada de la era de la información y el conocimiento, la perspectiva de las organizaciones cambió, dejaron de ser vistas como un conjunto de recursos humanos, tecnológicos, financieros y materiales que perseguían beneficios monetarios, generaban trabajo, y producían bienes y servicios, para ser consideradas desde otra óptica. En la actualidad, se enfocan las capacidades y talentos del capital humano para aprovechar las oportunidades y se piensa en una forma diferente de hacer las cosas, a través de una nueva dimensión humana en las empresas. Las organizaciones, se caracterizan por ser lucrativas, producen riquezas para todos sus integrantes e incentivan y favorecen la innovación, así como la adaptabilidad a los cambios. Así mismo, las organizaciones son flexibles a las transformaciones, lo cual les garantiza un crecimiento constante y la posibilidad de permanecer en el tiempo con su área de negocio.

En el ámbito organizacional, uno de los aspectos que hace que una empresa sea exitosa, es que estas reconocen el talento de sus integrantes y procuran desarrollarlo, pues entienden que ellos tienen metas ligadas al crecimiento del negocio y que serán remunerados de acuerdo con el cumplimiento de los objetivos establecidos para lograrlo.

Una de las formas que han encontrado las organizaciones para adaptarse a los constantes cambios en el mercado, y que están asociados a las tendencias en materia del talento humano, es la incorporación de sistemas de gestión basados en competencias, lo cual se apoya en que el talento que se ha integrado al capital intelectual de la organización, refleja competencias individuales, alto desempeño, compromiso, adaptabilidad, flexibilidad y cultura de excelencia. 
El desempeño laboral de sus empleados, está orientado hacia un servicio eficiente y eficaz para llevar a cabo las actividades de administración tributaria, pero es el componente humano el cual garantiza las funciones y la convivencia que se despliega.

La gestión del talento humano ha sido uno de los nortes de la institución y el aporte de capital intelectual ha simbolizado un pilar fundamental y de gran importancia, desde el cual se pretende lograr que los trabajadores alcancen su máximo desempeño sobre la base de sus habilidades, cualidades y destrezas, siendo considerados para potenciar su desarrollo. Sin embargo, se debe impulsar la capacitación del personal administrativo, ya que se registra debilidad en esta área y desconocimiento de la gestión del talento humano para un excelente desempeño laboral, así mismo se presenta resistencia al cambio, lo que trae consigo el desempeño poco efectivo e ineficiente en el desarrollo de sus funciones y tareas, limitando así el desenvolvimiento en sus habilidades y destrezas.

Entre los desarrollos más relevantes de esta investigación se encuentran las características personales, conductas y comportamientos que se desean cambiar en el individuo para que desarrolle un trabajo eficiente. En ese sentido, se evidencia una debilidad en el manejo de las herramientas de liderazgo que mejoran el trabajo de los funcionarios, por tal razón el objetivo planteado es el desarrollo del talento humano, mediante los líderes de gerencia en la administración tributaria dentro del cambio organizacional. A su vez, se busca concientizar a nivel empresarial sobre la optimización del tiempo y de los recursos, como del empleado en función de las competencias identificadas en el puesto de trabajo, y estrategias metodológicas para que los supervisores puedan llevar a cabo exitosamente las actividades de formación. Así, por último se debe seguir una planificación de las evaluaciones donde se vea reflejada la ejecución, evaluación y control para lograr con éxito la puesta en marcha de un nuevo sistema de gestión y desarrollo por competencias en el ámbito de formación.

En suma, el valor agregado de esta investigación a la organización lo constituye el hecho de que partirá del propio talento humano con el que cuenta para formar a otros, ya que serán los supervisores de las líneas tácticas y estratégicas, quienes formarán a sus subordinados. Lo cual tiene un indiscutible impacto positivo en el tema de asignación e inversión de recursos financieros a nivel de formación, desarrollo y talento humano. 


\section{ANTEGEDENTES DE LA INVESTIGACIÓN}

Para adentrarse en el desarrollo teórico y práctico de esta investigación, se realizó la consulta de trabajos que lo preceden, en los que se evidencia resultados que de alguna manera vinculan la formación en las organizaciones, como un modelo de gestión organizacional.

De acuerdo con el Observatorio de los Recursos Humanos (ORH), en el marco de transformación "nunca antes los líderes de este departamento habían experimentado tanta presión para alinearse con los directivos de las organizaciones, con el objetivo de apoyar el crecimiento y permitir que la compañía continúe siendo competitiva” (2020). Es tan imperativo tratar este tema que, de acuerdo con Bersin et al. (2017) "el $88 \%$ de los participantes en la encuesta de este año creen que la construcción de la organización del futuro es un asunto importante" (p. 19).

La aplicación de prácticas innovadoras derivadas de los cambios en las tendencias globales en esta área es muy relevante, pues los consumidores del mercado laboral a los que se hace referencia en la primera definición son los empleados, ya que no solo existen las demandas externas referentes al contexto, también están las internas que aluden a los propios empleados, cuyas necesidades cambian de acuerdo a los avances tecnológicos y a la sociedad, por lo que sus requerimientos deben ser satisfechos a la par con los del mercado. De acuerdo con Mercer (2017), recursos humanos "encontrará necesario desarrollar la capacidad de atraer, desarrollar y conservar el mejor talento en mercados emergentes" (p. 73).

Por otra parte, desde el 2013 Deloitte (2017) ha ejecutado investigaciones de directrices globales para el capital humano, en las cuales se afirma la importancia de dar inicio a los cambios en el mundo del trabajo, esta organización considera que los cambios y las brechas entre tecnología, individuos, empresas y políticas públicas está creando una oportunidad única para que los departamentos de RR. HH. le colaboren a los líderes y organizaciones a adaptarse a la tecnología, y también, ayudar a las personas a adaptarse a los nuevos modelos de trabajo y carreras.

Desde otra perspectiva se señala el antecedente de García y Sejias (2012), quienes realizaron un estudio denominado Estrategias de aprendizaje para entrenar facilitadores de proceso en el Banco del Caribe, en la Universidad Católica Andrés Bello para optar al título de Magíster en Educación. El estudio se enfoca en la iniciativa del Banco del Caribe por involucrar directamente a su recurso interno en las actividades de entrenamiento 
que coordina. La investigación consiste en crear una plantilla de facilitadores de procesos a nivel nacional, a fin de descentralizar y delegar la responsabilidad del entrenamiento a los gerentes de cada región.

De allí que el objetivo general de la investigación fue diseñar una guía de aprendizaje para entrenar de manera integral a la plantilla de facilitadores de procesos, previa selección del banco a fin de efectuar un desarrollo exitoso en el proceso enseñanzas-aprendizaje.

En estos antecedentes se demuestra que las investigaciones orientadas hacia el tema del desarrollo humano como base en la gerencia, es un tópico fundamental de interés mundial, ya que todas las organizaciones quieren alcanzar el éxito. Además, el éxito ofrece innumerables ventajas competitivas a las organizaciones y por tanto, cada día va en aumento. Lo anterior, es una clara muestra de que las organizaciones tienden a ser hoy en día organizaciones del conocimiento, que solo aprenden a través de individuos que se forman. Por ende, el entrenamiento y la capacidad son los medios para crear el compromiso de los empleados en el marco de un proceso de cambio organizacional y difundir conocimientos, promover su aplicación práctica en pro de la obtención de resultados concretos, es lo que genera los cambios necesarios para continuar compitiendo en el mercado. Educar es una actividad transformadora, transmitir conocimientos, desarrollar habilidades y facilitar ciertas actitudes, es habilitar a las personas para promover cambios en sí mismos y en su entorno.

\section{Algunas consideraciones en cuanto a tiempo del Ministerio de Hacienda con base en su función y delegaciones}

En el progreso de esta investigación se tomaron en consideración algunos aspectos relevantes relacionados al Ministerio de Hacienda y Función Pública, departamento de la Administración General del Estado encargado de la propuesta y ejecución de la política del Gobierno en las siguientes materias: hacienda pública; presupuestos y gastos; empresas públicas; gestión de los sistemas de financiación y cooperación con la administración autonómica y local; apoyo a las delegaciones y subdelegaciones del Gobierno; función pública; empleo público; formación de empleados públicos de reforma y organización de la Administración General del Estado; procedimientos e inspección de servicios; impulso de la administración electrónica; evaluación de políticas públicas, mejora de la gestión pública y la calidad de los servicios. Este departamento ha sido creado por Real Decreto 
1823/2011 del 21 diciembre, y se ha definido su estructura orgánica por los Reales Decretos 1829/2011 del 23 de diciembre; 1887/2011 del 30 de diciembre, y 256/2012 del 27 de enero.

En la actualidad, gracias al recorrido histórico de la institución, se puede observar su dinamismo en cuanto a la aceptación o adaptación a los cambios que sugiere la realidad socio-política y económica del país. Es un órgano moderno y dinámico que mira al futuro gracias a la inmensa experiencia que ha acumulado desde 1705 hasta nuestros días.

\section{APROXIMACIONES TEÓRICAS EN RELACIÓN AL TALENTO HUMANO, LIDERAZGO Y CAMBIO ORGANIZACIONAL}

En la actualidad, las organizaciones se ven sometidas a retos, desafíos y presiones a las cuales tienen que responder con alto grado de creatividad, realismo y responsabilidad. Los principales retos están dados por la dinámica de la aplicación de logros tecnológicos, la aparición y aceptación de los nuevos desafíos que cada vez presentan mayores restricciones de recursos humanos, materiales y financieros, mercados más agresivos y dinámicos en el ámbito internacional, el crecimiento de las demandas sociales y la revolución de la informática y comunicaciones.

Por esa razón, tras los significativos cambios socioeconómicos, cada vez más, las empresas modernas concuerdan en reconocer la significación que posee la dimensión humana de la empresa y la gestión del talento humano, igualando en grado de importancia a los aspectos económicos, financieros y tecnológicos.

Según Chiavenanto (2002), la gestión del talento humano es un área muy sensible a la mentalidad que predomina en las organizaciones. Es contingente y situacional al depender de aspectos como la cultura de cada organización, la estructura organizacional adoptada, los caracteres del contexto ambiental, el negocio de la organización, la tecnología utilizada, los procesos internos, entre otros procesos de relevancia.

Actualmente en el ámbito de la formación y desarrollo del talento humano de las organizaciones, se presenta como fin común lograr la competitividad de las organizaciones ante diversos factores condicionantes. Todos ellos exigen, de alguna forma, cambiar los enfoques tradicionales del tratamiento de este recurso, otorgándole el 
significado requerido por su aporte a los resultados de la empresa. El rasgo principal de esta nueva tendencia, es la incorporación de nuevos términos en el ámbito organizacional que tienen que ver con el liderazgo y la formación. Cualquier aporte que se realice en esta materia, implica pertinencia, importancia laboral y por ende, un aspecto social, pues las ideas que emanan del estudio de este tema, propician un nuevo estilo de aprendizaje y formación del talento humano, adaptado enteramente a las organizaciones que buscan sacar el mayor provecho a los trabajadores que conforman las instituciones, reconociendo una inversión mínima para ello.

A su vez, se considera la toma de consciencia en relación a la gestión y desarrollo del talento humano para líderes gerenciales, de modo que se garantiza el desarrollo de conductas, habilidades y destrezas que deseen formar en los trabajadores, a fin de contribuir con el nuevo modelo de gestión que destaca un talento humano altamente entrenado y calificado.

Así bien, en palabras de Schuler (1992), puede definirse como la gestión estratégica de los recursos humanos, a todas aquellas actividades que afectan el comportamiento de las personas a la hora de formular e implantar las estrategias de las empresas o las decisiones respecto a la orientación dada a los procesos de gestión de los recursos humanos que afectan al comportamiento de los individuos a mediano y largo plazo, tomando como referencia los factores internos, externos y de contexto a la organización.

Teniendo en cuenta lo planteado por el autor, se entiende que hay estrategias alternativas que pueden desarrollarse dentro del talento humano, pues su conocimiento constituye una herramienta teórica y metodológica para el diseño de diferentes estrategias en las cuales se puedan involucrar los empleados dándoles diferentes herramientas a los gerentes líderes que mejoren y optimicen los procesos en la institución, específicamente en la gerencia financiera administrativa tributaria, como respuesta a la constante búsqueda del mejoramiento continuo y de explorar nuevas formas de desarrollo del personal.

En ese orden de ideas, los gerentes deben ser líderes dedicados a crear confianza dentro de las organizaciones empresariales, ser el pilar fundamental que haga que todas las actividades se realicen de forma óptima. Y es desde este punto de vista de la gran responsabilidad de los gerentes líderes en las empresas, que deben desarrollar destrezas de liderazgos de forma automática y estar siempre en una constante 
innovación, capacitación de su persona y del personal que está a su cargo. En esa línea, Bennis (1985) afirma que el líder es el que comprende a la gente, a la acción y es quien transforma seguidores en líderes y puede convertirlos en agentes de cambio.

Las destrezas de liderazgo se identifican como habilidades o características clave determinantes en el éxito personal y profesional de las personas. En el ambiente laboral, existe un talento humano que debe cumplir diferentes labores según su puesto o desempeño dentro de la empresa, en tanto, el líder debe llegar a cada ser humano para que realice la labor de forma exitosa tomando en cuenta la opinión de RR. HH. para plantear las mejoras continuamente, adaptándose a los cambios.

Es así como esta investigación propone un marco referencial hacia una nueva metodología de trabajo dentro de la administración tributaria, basada en la premisa de mantenerse a un nivel competitivo dentro de un mercado organizacional cada vez más especializado y capacitado. Por ese motivo, se debe partir del talento humano con el que se cuenta, cultivar y cosechar las habilidades del trabador, una buena forma de comenzar a hacerlo es mantenerse siempre pensando en ser los mejores; ya sea que se trabaje con un modelo establecido o con uno innovador, será sin duda un nuevo estilo de formación interna de líderes en el ámbito organizacional, que buscar sacar de cada persona la excelencia y su verdadero potencial.

Es desde aquí que parte el cambio organizacional dentro de las empresas, según Chiavenato (2002). "El cambio organizacional es un conjunto de alteraciones estructurales y de comportamiento dentro de una organización" (p. 418), dos conceptos que se han venido difundiendo ampliamente en las últimas décadas en los ambientes académicos y empresariales, conocidos como desarrollo organizacional y la calidad total.

Ahora bien, si se analiza de forma práctica el aspecto académico, mientras más capacitación se dé dentro de la empresa, el recurso humano que se gesta es cada vez más óptimo, lo cual trae aspectos positivos para la mejora de la empresa y para el correcto desarrollo organizacional, tomando todos los aspectos fundamentales para su progreso. Como premisa fundamental está el liderazgo en las gerencias dentro del desarrollo del talento humano, para lograr un cambio organizacional, siendo estos postulados una triada completa para el éxito dentro de la administración tributaria. 


\section{MARCO METODOLÓGICO}

El diseño metodológico de la investigación se aplicó para medir el nivel de conocimiento de las variables de estudio: talento humano, líderes gerenciales, y cambio organizacional, y de este modo cumplir el objetivo general planteado, es decir, el desarrollo del talento humano por medio de los líderes de gerencia en la administración tributaria, dentro del cambio organizacional. Y a su vez, concientizar a nivel empresarial la importancia de la optimización del tiempo y de los recursos, así como del empleado en función de las competencias identificadas en su puesto de trabajo para tomar las decisiones correspondientes al desarrollo del talento humano, como actividades dentro del cambio organizacional que se realizaron para el logro del objetivo, y por ende, dar respuesta a uno de los procesos cambiantes dentro de un plano gerencial y empresarial. De esta manera, se concibe la evaluación desde el punto de vista de los 29 gerentes que están dentro del área de la administración tributaria, siendo una población finita en la cual se toma la muestra en su totalidad. Se planteó una metodología bajo un diseño evaluativo, apoyado en un diseño no experimental, con sustento de la investigación de campo, a nivel descriptivo, evaluativo expost y de corte cuantitativo.

A esta población de 29 gerentes se les aplicó un instrumento tipo cuestionario de escala Likert, para medir las siguientes variables de estudio: talento humano, líderes gerenciales y cambio organizacional.

Como se sabe, la investigación que nos ocupa está referida al desarrollo del talento humano para líderes de la gerencia financiera del servicio integrado de administración tributaria con el fin de lograr un cambio organizacional; para abordar dicha situación, se hace necesario estudiar a profundidad cada uno de los factores intervinientes en el contexto, para lograrlo se describen, registran, analizan e interpretan detalladamente las tareas y actividades asociadas en el hecho objeto de estudio.

Selltiz y Jahoda (citados por Ramírez, 1999), indican que una de las modalidades de investigación a nivel descriptivo tiene como objetivo: "La descripción, con mayor precisión, de las características de un determinado individuo, situaciones o grupos, con o sin especificación de hipótesis iniciales acerca la naturaleza de tales características" (p. 84).

La investigación se orienta en este nivel, sobre la base de un cambio en la visión del talento humano, como lo es la preparación de líderes gerenciales dentro del cambio organizacional, en la gerencia financiera administrativa tributaria. 


\section{ANÁLISIS E INTERPRETACIÓN DE LOS RESULTADOS}

El instrumento elaborado para la recolección de los datos fue el cuestionario, este se aplicó a la población total correspondiente al desarrollo del talento humano, compuesta por 29 gerentes del área financiera administrativa tributaria. La escala de medición considerada para dicho instrumento, se organizó en la siguiente ponderación del 1 al 5 , siendo 1-Muy bajo, 2-Bajo, 3-Regular, 4-Bueno, y 5-Excelente.

Tabla 1. Desarrollo del talento humano

\begin{tabular}{|c|c|c|c|c|c|}
\hline Componente 1 & $\begin{array}{c}1 \\
\begin{array}{c}\text { Muy bajo } \\
(\%)\end{array}\end{array}$ & $\begin{array}{c}2 \\
\text { Bajo } \\
(\%)\end{array}$ & $\begin{array}{c}3 \\
\text { Regular } \\
(\%)\end{array}$ & $\begin{array}{c}4 \\
\text { Bueno } \\
(\%)\end{array}$ & $\begin{array}{c}5 \\
\text { Excelente } \\
(\%) \\
\end{array}$ \\
\hline Conocimientos teóricos & & & 23,08 & 15,38 & 61,54 \\
\hline Conocimientos técnicos & & & & 30,77 & 69,23 \\
\hline Experiencia & & & & 30,77 & 69,23 \\
\hline Comunicación efectiva & & & & 53,85 & 46,15 \\
\hline Comunicación asertiva & & & 15,38 & 38,46 & 46,15 \\
\hline Habilidades de formación & 7,69 & 15,38 & 53,85 & 15,38 & 7,69 \\
\hline Estrategias metodológicas & & 15,38 & 23,08 & 30,77 & 30,77 \\
\hline Elabora actividades de formación & 15,38 & 30,77 & 30,77 & 15,38 & 7,69 \\
\hline Desarrollo de estrategias metodológicas & 7,69 & 53,85 & 23,08 & 7,69 & 7,69 \\
\hline
\end{tabular}

Fuente: elaboración propia.

\section{Interpretación}

En el componente 1, el porcentaje obtenido fue del 92,31\% del manejo y dominio cognitivo en los especialistas de las áreas teóricas, técnicas y de experiencia; en lo respecta al componente 2, señala en igual porcentaje el 92,31 \% de habilidades de comunicación; por su parte los componentes 3 y 4 hacen referencia a lo metodológico, presentando en el primero el 42,31 \% de habilidades y estrategias empleadas en procesos de formación y el siguiente el 19,23 \% referidos a la elaboración y desarrollo de estrategias metodológicas en actividades de formación en la organización en la gestión del talento humano. 
En esta primera sección, se refleja claramente que los gerentes siendo especialistas en las diversas áreas que integran la gerencia financiera administrativa, cuentan con aceptables niveles cognitivos y de comunicación, registran tener conocimiento sobre habilidades y estrategias para ser empleadas en encuentros formativos y de trabajo táctico, pero no saben cómo aplicarlo. De esta forma, se evidencia una notoria carencia o bajo dominio y manejo de estrategias para la elaboración y desarrollo de las actividades de formación en la organización de la gestión del talento humano.

Tabla 2. Líderes gerenciales

\begin{tabular}{|c|r|r|r|r|r|}
\hline Teóricas & \multicolumn{1}{|c|}{$\begin{array}{c}\mathbf{1} \\
\text { Muy bajo } \\
\mathbf{( \% )}\end{array}$} & \multicolumn{1}{c|}{$\begin{array}{c}\text { Bajo } \\
\mathbf{( \% )}\end{array}$} & $\begin{array}{c}\text { Regular } \\
\mathbf{( \% )}\end{array}$ & $\begin{array}{c}\text { B } \\
\text { Bueno } \\
\mathbf{( \% )}\end{array}$ & $\begin{array}{c}\text { Excelente } \\
\mathbf{( \% )}\end{array}$ \\
\hline Conocimientos del área & & & & 38,46 & 61,54 \\
\hline Teorías de aprendizaje & 15,38 & 15,38 & 53,85 & 7,69 & 7,69 \\
\hline Métodos de enseñanza & 7,69 & 7,69 & 69,23 & 7,69 & 7,69 \\
\hline $\begin{array}{c}\text { Planifica estrategias dentro de los } \\
\text { encuentros formativos }\end{array}$ & 23,08 & 38,46 & 23,08 & 7,69 & 7,69 \\
\hline $\begin{array}{c}\text { Organiza encuentros formativos } \\
\text { Actualización en el área }\end{array}$ & 15,38 & 38,46 & 15,38 & 23,08 & 7,69 \\
\hline Manejo de métodos de enseñanza & 23,08 & 38,46 & 30,77 & 23,08 & 69,23 \\
\hline Emplea métodos de enseñanza & 23,08 & 46,15 & 23,08 & & 7,69 \\
\hline
\end{tabular}

Fuente: elaboración propia.

Tabla 3. Cambio organizacional

\begin{tabular}{|c|c|c|c|c|c|}
\hline Metodología para el cambio & $\begin{array}{c}1 \\
\text { Muy bajo } \\
(\%)\end{array}$ & $\begin{array}{c}2 \\
\text { Bajo } \\
(\%)\end{array}$ & $\begin{array}{c}3 \\
\text { Regular } \\
(\%)\end{array}$ & $\begin{array}{c}4 \\
\text { Bueno } \\
(\%)\end{array}$ & $\begin{array}{c}5 \\
\text { Excelente } \\
(\%)\end{array}$ \\
\hline Planifica actividades formativas & 23,08 & 46,15 & 15,38 & 7,69 & 7,69 \\
\hline Utiliza recursos organizacionales & & 23,08 & 46,15 & 23,08 & 7,69 \\
\hline $\begin{array}{l}\text { Domina procesos } \\
\text { organizacionales }\end{array}$ & & & 30,77 & 23,08 & 46,15 \\
\hline $\begin{array}{l}\text { Desarrollo de habilidades por } \\
\text { experiencia }\end{array}$ & & 7,69 & 30,77 & 23,08 & 38,46 \\
\hline Manejo técnico & & & & 30,77 & 69,23 \\
\hline
\end{tabular}

Fuente: elaboración propia. 
Tabla 4. Concepción del cambio organizacional

\begin{tabular}{|c|c|c|c|c|c|}
\hline Sociales & $\begin{array}{c}1 \\
\text { Muy bajo } \\
(\%)\end{array}$ & $\begin{array}{c}2 \\
\text { Bajo } \\
(\%)\end{array}$ & $\begin{array}{c}3 \\
\text { Regular } \\
(\%)\end{array}$ & $\begin{array}{c}4 \\
\text { Bueno } \\
(\%)\end{array}$ & $\begin{array}{c}5 \\
\text { Excelente } \\
(\%)\end{array}$ \\
\hline Procesos de grupo & & & 15,38 & 53,85 & 30,77 \\
\hline Trabajo en equipo & & & 7,69 & 38,46 & 53,85 \\
\hline Relaciones interpersonales & & & & 30,77 & 69,23 \\
\hline Liderazgo & & & 15,38 & 15,38 & 69,23 \\
\hline Iniciativa & & & & 23,08 & 76,92 \\
\hline Autocontrol & & & & 30,77 & 69,23 \\
\hline Creatividad & & & & 30,77 & 69,23 \\
\hline Comunicación & & & & 38,46 & 61,54 \\
\hline Flexibilidad & & & & 46,15 & 53,85 \\
\hline $\begin{array}{l}\text { Compromiso con la } \\
\text { organización }\end{array}$ & & & & 23,08 & 76,92 \\
\hline Orientación al funcionario & & & & 23,08 & 76,92 \\
\hline
\end{tabular}

Fuente: elaboración propia.

\section{Interpretación}

El 40,66 \% de los gerentes mantiene sólidos conocimientos teóricos y constante actualización en su área específica de trabajo.

En lo referente a las competencias metodológicas para el cambio organizacional, las habilidades desarrolladas por experiencia en actividades formativas, relacionadas con la planificación, dominio y manejo, arrojaron como resultado un 55,38 \%, lo cual indica que basados en los conocimientos adquiridos por la práctica laboral y el dominio de procesos, los supervisores en las áreas ejecutan actividades de formación con debilidades en herramientas y estrategias metodológicas.

Por otro lado, en las competencias sociales, la población analizada reconoce tener altos niveles competitivos, registrando un 97,08\% de estos, índice de excelencia que pone en evidencia las capacidades, destrezas y habilidades que posee la línea táctica y estratégica de la gerencia financiera administrativa. En resumen, los supervisores indiscutiblemente son expertos en su área y tienen la disposición para llevar a cabo procesos 
en el equipo, simplemente no manejan estrategias de formación para ser entes multiplicadores del conocimiento.

Ahora bien, a partir del análisis anterior ha sido posible dar cumplimiento al objetivo general planteado, y en referencia a las tres variables de estudio propuestas, se tiene lo siguiente:

- En el contexto de formación del talento humano: los resultados arrojados por la aplicación del instrumento determinaron que aun cuando la institución posee conocimientos teóricos sobre su área de especialización y son expertos en ella, no poseen las habilidades y estrategias pedagógicas para llevar a cabo actividades de formación con su propio equipo de trabajo.

- En el ámbito de los líderes de gerencia: es evidente el total conocimiento e identidad de los gerentes de la organización con la filosofía de la institución, lo cual permite incorporar nuevas figuras asociadas a la formación como elemento inequívoco del mejoramiento continuo.

- En el contexto del cambio organizacional: se entiende que los gerentes poseen las competencias teóricas y conceptuales asociadas a su área de conocimiento y se actualizan constantemente en ella. Debido a su condición han estado expuestos a liderar a sus equipos de trabajo por cierto tiempo, lo cual de alguna forma les permite poseer habilidades en el manejo de equipos, sin embargo, a través de la interpretación de los resultados, es evidente que ellos no tienen el manejo de las teorías básicas de aprendizaje y por tanto, no emplean adecuados métodos de enseñanza, es decir, que no cuentan con las competencias metodológicas requeridas asociadas a la planificación de actividades formativas y al dominio de estrategias de enseñanza-aprendizaje, para llevar a cabo actividades de formación bajo la figura de facilitadores gerenciales.

\section{CONGLUSIONES}

Tras haber identificado los elementos que subyacen por competencias, enfocándolos a los resultados obtenidos de la aplicación del instrumento de medición en la institución, se determinó lo siguiente:

1. El recurso humano está alineado con el enfoque de gestión por competencias: parte de una filosofía profundamente 
comprometida con su entorno y con sus líderes, se enfoca en ser una institución competitiva, eficiente, que genere confianza, seguridad y rentabilidad al Estado, para que sea reconocida como una organización que se mantiene a la vanguardia en cuanto a la calidad de sus servicios, confianza, seguridad y cultura tributaria que se traduce en beneficios para el Estado. Además, ser una institución que apoye de manera integral a sus gerentes, para disminuir así los costos financieros, brindando asesoría e información oportuna y confiable para mantener una comunicación efectiva y asertiva mediante el uso eficiente de los recursos humanos, financieros y materiales, en el marco de la integridad, trabajo en equipo, alto desempeño y aprendizaje permanente.

2. Lo que se desea mejorar en los gerentes para que desarrollen un trabajo aún más eficiente es el desempeño, la conducta y el comportamiento. La organización cuenta con un valioso talento dentro de su nómina de gerentes, que se mantienen en ella haciendo carrera por muchos años, los cuales han sido objeto de movilizaciones internas, (ascensos o traslados) o a otras zonas del país. Muchos de ellos han puesto en evidencia su total capacidad, habilidades y destrezas a la hora de realizar sus funciones, se han convertido en expertos de su área y por tanto, pueden ser partícipes de la formación de sus propios compañeros de trabajo o subordinados.

3. Los resultados indicados por el instrumento aplicado a la población, pone en evidencia que los gerentes poseen un adecuado nivel teórico y están facultados para el manejo de equipo, sin embargo, también muestra su debilidad en cuanto al manejo de herramientas y estrategias metodológicas para que puedan llevar a cabo exitosamente actividades de formación. Es posible que en este momento las realicen, pero lo que se plantea en este proyecto es "el cómo" lo hacen. Es preciso mejorar la aplicación de estrategias adecuadas a los que serán los líderes del mañana en la administración tributaria nacional, es el impacto que causa en los ámbitos de desarrollo continuo, rendimiento, productividad y temas que están asociados al aprendizaje y desempeño.

4. Se debe seguir una metodología de planificación, ejecución, evaluación y control para lograr con éxito la gestión por competencias en el ámbito de la formación laboral interna. Por 
tanto, se hace preciso detallar lo concerniente al desarrollo de gerentes y recursos humanos bajo la perspectiva de competencias, la calidad de líderes internos y preparación en la gerencia financiera administrativa tributaria, de lo cual dependerá el éxito de la formación de sus gerentes. Por este motivo, es evidente que más allá de considerar a una persona para que cumpla la función de líder gerencial, se deberán aplicar ciertos criterios de selección teniendo en cuenta no solo sus cualidades técnicas y conocimiento sobre una materia en particular, sino también sus cualidades personales, pues su conocimiento puede constituir una herramienta teórica y metodológica valiosa para el diseño o perfeccionamiento de algún proceso de formación.

Dicho lo anterior, un gerente tiene que ser una persona capaz de crear aportes positivos dentro de una la administración tributaria, debe contar con características que le permitan ser un líder, dar seguridad para ayudar a un conjunto de recursos humanos a lograr metas en un corto, mediano y largo plazo, debe estar dispuesto a planificar cambios en tiempos planteados, tener claros los retos de la globalización y la tecnología, sin alejarse del talento humano. Así mismo, tener sentido de pertenencia y curiosidad por el aprendizaje continuo, debido a que él es quien debe estar siempre capacitándose, estudiando y siendo motivado para estar al nivel de todos los cambios que se pueden visionar o crear.

En la actualidad, un gerente debe poseer una gran capacidad de acumular conocimiento, habilidades y destrezas, capacitarse en cuanto a la información, mantener un nivel jerárquico marcando la pauta en cuanto a los valores, la responsabilidad, la calidad humana como persona íntegra, la administración empresarial, y a su vez, la gerencia humana. Desde esta premisa es importante que marcar un equilibrio, porque el proceso de los cambios organizacionales va de forma constante, que solo una decidida preparación, es la única manera que puede marcar la durabilidad dentro de un puesto gerencia. Es primordial que el gerente tenga una visión con ideas clara sobre el proceder de su función primordial como ser humano, como líder, manejar los diferentes esquemas económicos, sociales y gubernamentales. Él debe saber la fórmula de cómo llegar a una meta empresarial y cómo se puede superar en muy cortos plazos, sin descuidar nunca a los empleados que son el recurso humano más importante dentro de una organización.

En cuanto al talento humano, el gerente siempre debe motivar, escuchar, observar las cualidades y fortalezas de cada uno de los seres 
humanos que coexisten en su espacio de trabajo, para así sacar lo mejor de ellos.

Los requerimientos de la actualidad demandan la formación de los recursos humanos y los gerentes guías en su ejercicio. Conjuntamente, necesita tener un desarrollo multidisciplinario con preparaciones científicas, técnicas, económicas, productivas y humanísticas; necesitan de todas las aptitudes y cualidades que le den el paso del liderar un conjunto de trabajadores para alcanzar el aumento del desarrollo, fabricación y capacidad en estructura copartícipe.

En ese orden de ideas, un punto crucial dentro del desarrollo actual de la gerencia y el recurso humano de la administración tributaria, se concentra en el desarrollo al máximo de las capacidades humanas de los trabajadores, para así poder llegar al éxito dentro del campo de la competencia diaria.

Para lograr satisfacer el trabajador al máximo, a diario debe existir un estímulo para su autoestima, es el modo de ubicarlo en su espacio y valorarlo. También es importante lograr que el empleado se sienta capaz de lograr las tareas con la suficiente eficacia y que crea en él, para así lograr sentirse bien y satisfecho. Con autoeficacia y autoestima será más fácil que un hombre sea más fructífero, y que esto repercuta positivamente en la productividad del componente humanitario.

Por último, respecto al proceso de capacitación, motivación del recurso humano y los líderes gerenciales, estos son la base de los cambios organizacionales que no deben dejarse al azar, ni a la inercia de la costumbre, menos a la improvisación, deben planificarse adecuadamente; por ello la intervención del talento humano es indiscutible. Así mismo, se plantea como punto importante buscar cuál es la percepción de la motivación ante el cambio organizacional, de manera que se sepa en qué fase o etapa se encuentra.

\section{REFERENCIAS}

Bennis, W. G., Nanus, B., \& Hoyos, E. (1985). Líderes: las cuatro claves del liderazgo eficaz. Norma.

Bersin, J., McDowell, T., Rahnema, A., \& Durme, Y. (2017). La Organización del Futuro. Chiavenato, I. (2002). Gestión del talento humano. McGraw-Hill.

Deloitte. (2017). Global Human Capital Trends report reflects seismic changes in the world of business. This new era, often called the Fourth Industrial Revolution. https:// 
www2.deloitte.com/co/es/pages/human-capital/articles/introductionhuman-capital-trends1.html

García, \& Sejias, F. (2012). Estrategias de aprendizaje para entrenar facilitadores de proceso en el Banco del Caribe [Tesis de pregrado]. Universidad Católica Andrés Bello.

Llegando ahora. (s.f). Tendencias Globales en Capital Humano, (5), 19- 24.

Mercer. (2017). Mercer's Global Talent Trends Study. https://www.mercer.com/ourthinking/career/global-talent-hr-trends.html

Observatorio de Recursos Humanos (2020). La gestión del talento y el futuro de los recursos humanos para 2020. https://www.observatoriorh.com/gestion/lagestion-del-talento-futuro-los-recursos-humanos-2020.html

Ramírez, T. (1999). Cómo hacer un proyecto de investigación. Editorial Panapo.

Schuler, R. S. (1992). Strategic Human Resource Management: Linking People with the Needs of the Business. Organizational Dynamics, 21(1) 18-32. https:// doi.org/10.1016/0090-2616(92)90083-Y 